\title{
Characterizing 5-methylcytosine in the mammalian epitranscriptome
}

\author{
Shobbir Hussain ${ }^{*}{ }^{+}$, Jelena Aleksic ${ }^{\dagger}$, Sandra Blanco, Sabine Dietmann and Michaela Frye
}

\begin{abstract}
The post-transcriptional modification 5-methylcytosine $\left(\mathrm{m}^{5} \mathrm{C}\right)$ occurs in a wide range of coding and non-coding RNAs. We describe transcriptome-wide approaches to capture the global $\mathrm{m}^{5} \mathrm{C}$ RNA methylome. We also discuss the potential functions of $\mathrm{m}^{5} \mathrm{C}$ in RNA and compare them to 6-methyladenosine modifications.
\end{abstract}

\section{Introduction}

Post-transcriptional changes in RNA processing are essential regulators for most, if not all, cellular responses. Although RNA modifications are more prevalent and diverse in their chemical nature than DNA modifications [1], our knowledge of their occurrence and function in RNA is generally limited. There are approximately 150 known ribonucleoside modifications: most of them have been found in tRNA and rRNA, but some occur in mRNA [1-4]. Post-transcriptional modifications are highly likely to add complexity to RNA-mediated functions.

The advent of next-generation sequencing (NGS) tools has enabled the identification of RNA modifications both globally and in a substrate-specific manner. 6Methyladenosine $\left(\mathrm{m}^{6} \mathrm{~A}\right)$ was the first modification to be characterized, and is now known to be present in several types of RNA and, most notably, is highly enriched around stop codons in many mRNAs [2-4].

Another known modification in RNA is 5-methylcytosine $\left(\mathrm{m}^{5} \mathrm{C}\right)$. Although $\mathrm{m}^{5} \mathrm{C}$ is a well-characterized modification in DNA, its precise regulatory functions in RNA remain unclear $[5,6]$. Until recently, the detection of RNA methylation involved the digestion of highly purified RNA followed by separation techniques, such as high performance liquid chromatography (HPLC) and mass spectrometry, which only allowed the identification of $\mathrm{m}^{5} \mathrm{C}$ in stable and highly

\footnotetext{
*Correspondence: sh465@cam.ac.uk; mf364@cam.ac.uk

${ }^{\dagger}$ Equal contributors

Wellcome Trust - Medical Research Council Cambridge Stem Cell Institute, University of Cambridge, Tennis Court Road, Cambridge CB2 1QR, UK
}

abundant tRNAs and rRNAs [7-10]. Labeling techniques with ${ }^{3} \mathrm{H}$ in living cells allowed detection of $\mathrm{m}^{5} \mathrm{C}$ in mRNA and viral RNAs [11,12]. However, the deposition of $\mathrm{m}^{5} \mathrm{C}$ into mRNA remained controversial [13-17]. The recent advances in high-throughput techniques, combined with NGS, have renewed interest in the field, and have led to the identification of $\mathrm{m}^{5} \mathrm{C}$ as a widespread modification in coding and non-coding (nc)RNAs [18].

A fraction of these RNAs were found to be specifically methylated by the RNA methylase NSun2, including mRNAs, ncRNAs and several tRNAs [18]. NSun2 had been previously shown to methylate tRNAs at various positions [19-22]. Additional NSun2-methylated coding and ncRNAs were identified in two studies published in 2013 that used customized RNA immunoprecipitation approaches followed by NGS [23,24].

The regulatory functions of $\mathrm{m}^{5} \mathrm{C}$ modifications in RNA are still not fully understood. In tRNAs, in vitro cytosine- 5 methylation can affect $\mathrm{Mg}^{2+}$ binding to tRNA molecules, which in turn influences the anticodon stem loop conformation and stabilizes the secondary structure $[25,26]$. Cytosine-5 methylation alone, or in combination with other nonessential tRNA modifications, can also protect from degradation or cleavage [22,27-29]. In rRNA, $\mathrm{m}^{5} \mathrm{C}$ is thought to play a role in translation [30]. Synthetic cytosine-5 methylated mRNAs exhibit increased stability, and loss of methylation in the 3' UTR of p16 has been reported to reduce its stability [31,32].

The biological functions of tRNA $\mathrm{m}^{5} \mathrm{C}$-methylation are linked to the regulation of protein translation in stress pathways and tissue differentiation in yeast, Drosophila, fish and mouse [19,22,29,33-36]. Mutations in the NSUN2 gene in humans cause an autosomal recessive syndrome characterized by intellectual disability, skin disorders and growth retardation [37-40]. These findings, together with studies carried out in NSun2-deficient mice and cell lines, suggest a wide-ranging role for $\mathrm{m}^{5} \mathrm{C}$ modifications in RNA, including cellular signaling, tissue development and differentiation, and cancer [19,21,22,36,41-43]. 
In this review, we compare the current methods to identify $\mathrm{m}^{5} \mathrm{C}$ in the mammalian transcriptome with a particular focus on NSun2-mediated methylation. We further discuss the potential of these initial studies to comprehensively determine the global but enzyme-specific cytosine- 5 RNA methylome. In addition, we compare studies focusing on $\mathrm{m}^{6} \mathrm{~A}$ and $\mathrm{m}^{5} \mathrm{C}$ modifications and consider the likely benefits of characterizing and elucidating the functions of the mammalian epitranscriptome.

\section{Mapping cytosine- 5 methylation in the mammalian transcriptome}

Previous methods for detecting $\mathrm{m}^{5} \mathrm{C}$ in RNA have required exceedingly high amounts of RNA and only reproducibly identified methylated sites in highly abundant RNAs, such as tRNAs and rRNAs. Over the last four years, NGS has allowed researchers to successfully develop more sensitive techniques. Bisulfite sequencing for the detection of $\mathrm{m}^{5} \mathrm{C}$ in RNA was first described in 2009 [44]. In 2012, the method was combined with NGS and provided the first transcriptome-wide view of the human cytosine-5 RNA methylome (Figure 1A) [18]. Earlier this year, three independent studies used RNA immunoprecipitation (RIP) followed by deep sequencing to detect $\mathrm{m}^{5} \mathrm{C}$ globally (Figures $\left.1 \mathrm{~B}-\mathrm{D}\right)[23,24,45]$. Khoddami and Cairns, and our own study [24], developed similar but technically distinct approaches to detect enzyme-specific deposition of $\mathrm{m}^{5} \mathrm{C}$ : 5-azacytidine-mediated RNA immunoprecipitation (Aza-IP) (Figure 1C) [23] and methylation-individual nucleotide resolution crosslinking immmunoprecipitation (miCLIP) (Figure 1D) [24]. Both Aza-IP and miCLIP rely on covalent bond formation between the RNA methylase and substrate but differ in the way in which the stable covalent bond formation is achieved. We now describe these techniques in detail and discuss their specific merits and disadvantages.

\section{Bisulfite sequencing}

Bisulfite sequencing, a method based on the chemical deamination of cytosines and originally developed to detect $\mathrm{m}^{5} \mathrm{C}$ in DNA, was previously adapted for use with RNA (Figure 1A) [44]. The technique is based on the differential chemical reactivity of $\mathrm{m}^{5} \mathrm{C}$ compared with cytosine. Sodium bisulfite causes the deamination of unmethylated cytosines into uridines in single-stranded DNA or RNA, while $\mathrm{m}^{5} \mathrm{C}$ remains unconverted [6]. A crucial parameter is the fraction of cytosine that is converted to uridine, but a high conversion rate is only achieved by prolonged incubation under consecutive acidic and alkaline conditions, which also causes RNA degradation. This degradation can compromise the

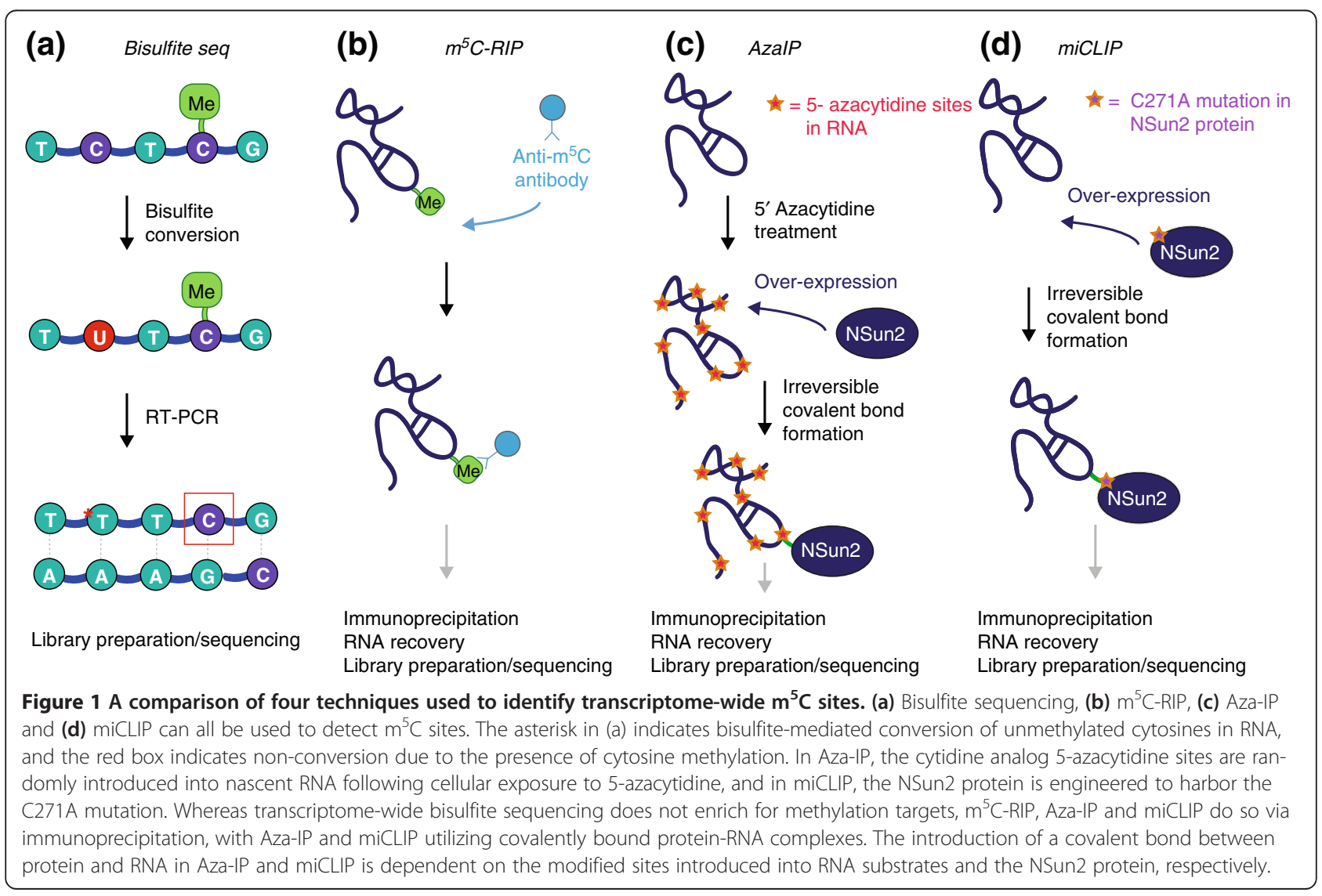


subsequent reverse transcription and PCR amplification steps $[18,46]$.

Using this approach, the occurrence of $\mathrm{m}^{5} \mathrm{C}$ can be reproducibly and quantitatively detected in tRNA and rRNA [44]. Coupling bisulfite conversion of RNA with NGS has led to detection of $\mathrm{m}^{5} \mathrm{C}$ in coding and noncoding RNAs, in addition to tRNAs $[18,45]$. Squires et al. [18] used polyA-enriched RNA and identified $\mathrm{m}^{5} \mathrm{C}$ as a widespread modification in coding and non-coding RNAs.

\section{$\mathrm{m}^{5} \mathrm{C}$-RNA immunoprecipitation}

In contrast to Squires et al., bisulfite sequencing of sizeselected mRNA was used by Edelheit et al. [45] to avoid tRNA and rRNA contamination from the prokaryote Sulfolobus solfataricus and, despite covering most coding genes, this approach detected low methylation levels in only 15 coding genes. However, all $\mathrm{m}^{5} \mathrm{C}$ sites were confirmed by RIP using a monoclonal antibody raised against $\mathrm{m}^{5} \mathrm{C}\left(\mathrm{m}^{5} \mathrm{C}\right.$-RIP) (Figure $\left.1 \mathrm{~B}\right)$ [45]. $\mathrm{m}^{5} \mathrm{C}$-RIP has not been performed in mammalian cells as yet.

\section{5-Azacytidine-mediated RNA immunoprecipitation}

Aza-IP exploits the formation of a covalent bond between RNA and specific RNA methylases (Figure 1C) [23]. Both DNA and RNA $\mathrm{m}^{5} \mathrm{C}$ methyltransferases form a temporary covalent bond between the catalytic cysteine in the enzymatic active site and carbon 6 of the targeted cytosine. This catalytic intermediate complex is later resolved to release the methylated cytosine at carbon 5 and to regenerate the free enzyme $[47,48]$. In the first step of the Aza-IP method, 5-azacytidine, a cytidine analog, is incorporated into nascent RNA molecules by RNA polymerases (Figure 1C) [23,49]. The only structural difference between cytidine and 5-azacytidine is a nitrogen substitution at carbon 5 that inhibits the release of the methylated RNA from its enzyme.

In a study by Khoddami and Cairns [23], this covalently bound protein-RNA complex was immunoprecipitated for the RNA methylases NSun2 and Dnmt2. The identification of the precise cytosine targeted by the enzymes was possible due to a specific transversion of cytosine to guanosine only at Dnmt2- and NSun2methylated sites. Although it is unclear why and how this conversion occurs, it has been confirmed that there are Dnmt2-dependent $\mathrm{m}^{5} \mathrm{C}$ sites in the three tRNAs Asp, Gly and Val $[22,23,29]$. Putative novel targets for Dnmt2 were the type I cytokeratin KRT18 mRNA and KRT18 pseudogene mRNA [23]. NSun2-dependent methylation sites occurred in tRNAs as well as several other ncRNAs. Although a potential site in the Src homology 2 domain containing $\mathrm{F}$ (SHF) mRNA was identified, statistically significant sites in other mRNAs were absent [23].

\section{Methylation-individual nucleotide resolution crosslinking immmunoprecipitation}

In an alternative approach, we utilized covalently bound catalytic intermediate RNA-protein complexes to identify methylation substrates of NSun2 (Figure 1D). NSun2 releases methylated RNA from the enzyme-substrate complex through a highly conserved cysteine residue in the catalytic domain [50,51]. Mutating this cysteine to alanine results in a covalently bound stable RNA-protein complex $[41,50,51]$. In the human NSun2 protein, this cysteine is represented by $\mathrm{C} 271$ and the mutation to alanine $(\mathrm{C} 271 \mathrm{~A})$ allowed capturing of stable NSun2-RNA complexes [24]. Combined with a customized individualnucleotide resolution cross-linking immunoprecipitation (iCLIP) approach where the UV-crosslinking step is omitted, and followed by NGS, we named this method methylation-iCLIP (miCLIP). This approach identified NSun2-mediated cytosine-5 methylation at the nucleotide resolution [24,52]. miCLIP not only confirmed known tRNA and non-tRNA methylation sites of NSun2, but also identified novel sites in coding and non-coding RNAs [24].

\section{Challenges of detecting site-specific 5-methylcytosines}

Of the four system-wide techniques that have been used to identify $\mathrm{m}^{5} \mathrm{C}$ sites in the epitranscriptome (Figure 1) $[18,23,24,45]$, RNA bisulfite sequencing remains the gold standard. This is because it directly detects the enzymatic conversion of cytosines to 5-methylcytosines in RNA. However, RNA bisulfite sequencing has at least three major practical disadvantages: (1) cytosines are resistant to conversion when in double-stranded RNA and several modifications other than $\mathrm{m}^{5} \mathrm{C}$ inhibit the $\mathrm{C}$ to $\mathrm{U}$ conversion, leading to false positives [53]; (2) the chemical conversion of the nucleotides leads to degradation of the RNA and requires extreme deep sequencing to reveal methylated RNAs of low abundance; and (3) it reveals no information about substrate specificity when, for instance, specific RNA methylases are knocked out. For instance, it cannot distinguish direct from indirect targets or capture the cross-reaction of additional RNA methylases.

$\mathrm{m}^{5} \mathrm{C}$-RIP, Aza-IP and miCLIP do not rely on chemical base conversion and avoid harsh chemical and thermal conditions that can lead to the degradation of target RNA. They also enrich for RNA substrates during the immunoprecipitation step. Therefore, RNAs with lower abundances can also be captured with standard deep sequencing protocols.

A key future goal in the $\mathrm{m}^{5} \mathrm{C}$ epitranscriptomics field is to gain a methylase-specific view of the mammalian $\mathrm{m}^{5} \mathrm{C}$ RNA methylome. The $\mathrm{m}^{5} \mathrm{C}$-RIP approach has yet to be performed in a mammalian system and, like bisulfite 
sequencing, analysis of enzyme-specific $\mathrm{m}^{5} \mathrm{C}$ modification requires genetic deletion or knockdown of the RNA methylase being investigated. Aza-IP and miCLIP not only allow detection of enzyme-specific methylation, but also have the advantage that the covalently bound methylaseRNA complexes can be purified under higher stringency conditions, thus reducing the background of nonspecifically bound RNAs.

The mechanism of cytosine-5 RNA methylation is highly conserved, and both Aza-IP and miCLIP are applicable, in principle, for most, if not all, $\mathrm{m}^{5} \mathrm{C}$ RNA methylases. Aza-IP relies on the chemical incorporation of 5-azacytidine into the entire transcriptome prior to isolation of protein-RNA complexes, which may compromise RNA stability and integrity. In addition, the analog will be incorporated into DNA and may alter transcriptional processes. Although Aza-IP was developed using overexpressed NSun2 and Dnmt2 proteins, it can in principle be performed on the endogenous methylases using the appropriate antibodies. In contrast, miCLIP depends on the overexpression of the mutant methylase protein, yet it does not involve any chemical modification of RNA, thus offering a distinct advantage. Since miCLIP only utilizes the last step of the catalytic process, in which the methylated substrate is released from the enzyme, it does not affect the methyl-transfer step, or the endogenous RNA structure, stability and integrity.

Particular consideration should also be given to the best bioinformatics approach to analyze different $\mathrm{m}^{5} \mathrm{C}$ sites. For instance, mapping the methylation sites in tRNAs is particularly challenging because of their abundance and repetitive nature [54,55]. One possibility is to only use uniquely mapping reads, which may result in the omission of methylation sites in particularly repetitive tRNA sequences to allow for more accurate mapping. However, since tRNAs are highly repetitive sequences in the genome, this often leads to discarding a large proportion of the available data. Another strategy is to use uniquely mapping reads to simulate the distribution of reads in a given area, thereby assigning multiple mapping reads proportionally. Mapping $\mathrm{m}^{5} \mathrm{C}$ sites obtained by bisulfite sequencing is hampered by the bisulfite conversion itself and RNA degradation, which may alter the base content.

AzaIP also presents some difficulties for identifying the complete set of methylation sites - a large number of targets often do not contain $\mathrm{C}$ to $\mathrm{G}$ transversions, and so the methylation sites in those targets are not mapped. Mapping methylation sites in miCLIP, on the other hand, does not depend on such artificial modifications, but rather on the stalling of the reverse transcriptase at the cross-link/ methylation site during the reverse transcription step. This phenomenon was utilized to efficiently map methylation sites.
While all four methods have their individual drawbacks, together they have the power to reliably detect $\mathrm{m}^{5} \mathrm{C}$ modifications. We will now turn our attention to comparing the cytosine- 5 methylated RNAs that have been identified in the mammalian transcriptome using the different techniques.

\section{A comparison of RNA $\mathrm{m}^{5} \mathrm{C}$ methylation sites identified by different approaches}

To better understand the ability of the different techniques to detect $\mathrm{m}^{5} \mathrm{C}$, we compared the data produced by the studies focused on the mammalian system $[18,23,24]$. Data used for the comparison are available in Additional file 1 . We compared global cytosine-5 methylation sites identified by RNA bisulfite sequencing with NSun2-dependent RNA methylation identified by AzaIP and our own developed method miCLIP (Figure 2) $[18,23,24]$. Each of the three studies identified $\mathrm{m}^{5} \mathrm{C}$ sites at nucleotide resolution. RNA bisulfite sequencing enriched for the mRNA fraction, identifying about $10,500 \mathrm{~m}^{5} \mathrm{C}$ sites [18]. The finding that $\mathrm{m}^{5} \mathrm{C}$ was a common modification in protein coding RNAs is surprising (Figure 2A) because cytosine-5 methylation has not yet been detected in mRNAs using alternative approaches such as mass spectrometry [1]. NSun2-specific Aza-IP [23] and miCLIP [24] using total RNA determined about 600 and 1,100 sites, respectively, suggesting that less than $10 \%$ of the methylome is directly dependent on NSun2. Most RNAs identified by both Aza-IP and miCLIP corresponded to tRNAs (Figure 2A), where approximately one-third of the tRNA methylation sites were commonly identified by both methods (Figure 2B; tRNA). In addition to tRNAs, all three studies identified $\mathrm{m}^{5} \mathrm{C}$ in non-coding RNAs. While bisulfite sequencing and miCLIP also identified several protein coding RNAs, only one statistically significant protein coding RNA was identified by Aza-IP. Aza-IP also detected additional rRNAs (Figure $2 \mathrm{~A}$ ). There are ten non-tRNA $\mathrm{m}^{5} \mathrm{C}$ sites found in at least two of the three studies, so these can be said to be true NSun2 non-tRNA targets with high confidence (Figure 2C).

The degree of overlap between the three studies was surprisingly low, and this may be due to several technical differences, as well as the use of different cell lines. Despite this low degree of overlap, the analyses found identical $\mathrm{m}^{5} \mathrm{C}$ sites in tRNAs and non-tRNAs and confirmed NSun2-dependent methylation sites in several ncRNAs. Our comparison further identified methylated cytosines in mRNAs commonly found in at least two of the three studies. However, we noted that these mRNA methylation sites often shared a genomic location with tRNA genes (see section below). Further optimization of the approaches is clearly required to achieve a 
(a)

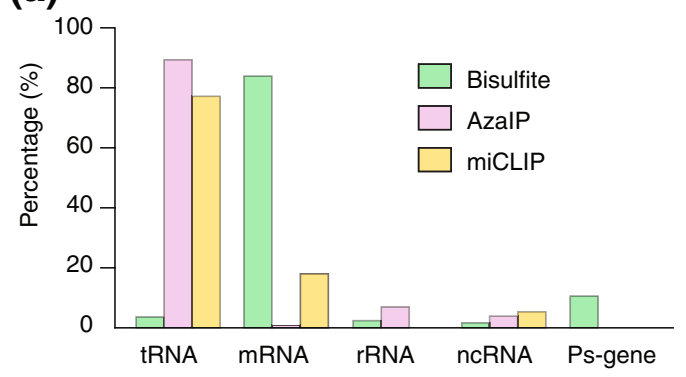

(b)
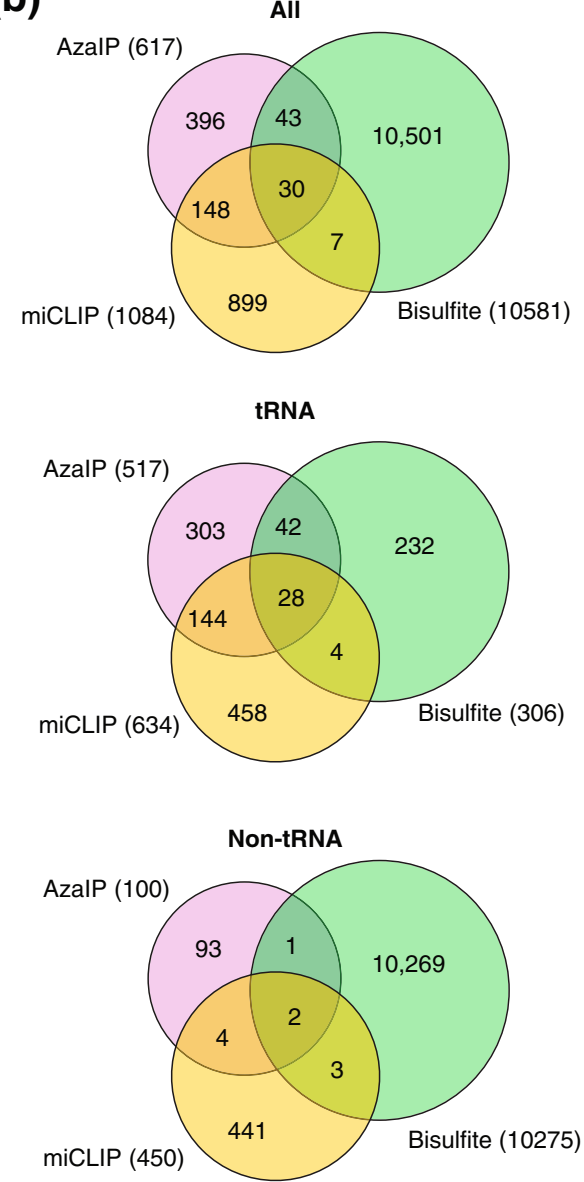

(c)

\section{All three studies}

\section{vtRNA1-1}

chr5: 140,090,929 ncRNA

$\mathrm{RPPH} 1$

chr14: 20,811,393 ncRNA (d)

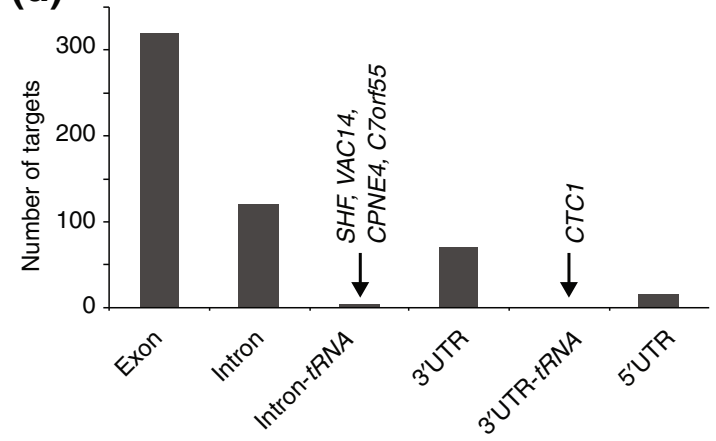

(e)

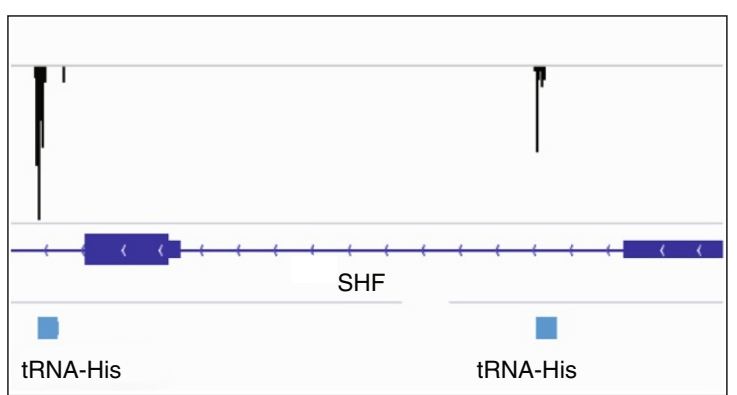

(f)

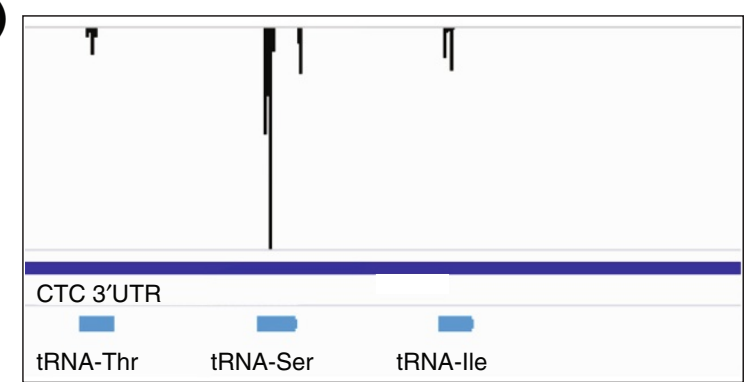

(g)

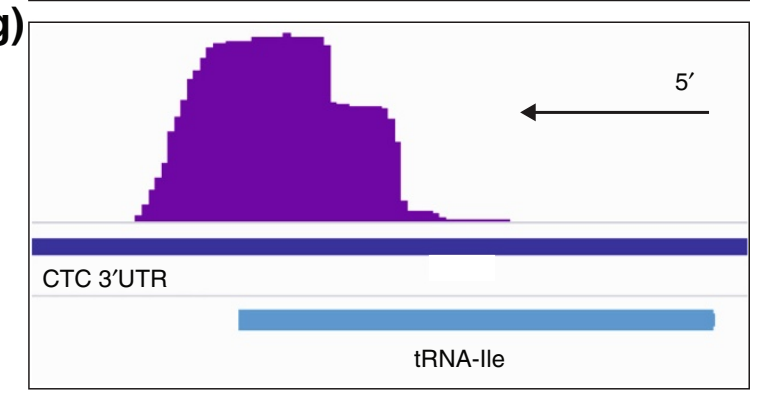

miCLIP/bisulfite

NSUN2

chr5: 6,604,788 mRNA

CTC1*

chr17: 8,129,578 mRNA

H3F3B

chr17: 73,774,027 mRNA
AzalP/miCLIP

5S rRNA

chr6: $4,428,287 \quad$ rRNA

vtRNA1-3

chr5: 140,105,758 ncRNA

vtRNA1-3

chr5: 140,105,770 ncRNA

SHF* $^{*}$

chr15: 45,492,612 mRNA

Figure 2 (See legend on next page.) 
(See figure on previous page.)

Figure $2 \mathrm{~A}$ comparison of $\mathrm{m}^{5} \mathrm{C}$ target sites identified by three transcriptome-wide approaches (Additional file 1) [18,23,24]. The bisulfite study identifies the global pattern of $\mathrm{m}^{5} \mathrm{C}$ methylation in the genome, while the AzalP and miCLIP data shown focus specifically on Nsun2mediated methylation. (a) Types of transcripts found to be methylated by each technique. (b) Comparison of the genomic locations of individual methylation sites found by each of the three approaches. The Venn diagrams show the overlap between all sites (upper panel), the sites that map to tRNAs (middle panel), and non-tRNA sites (lower panel). (c) Non-tRNA target sites found in at least two of the three studies, which represent high-confidence sites. The genomic location (hg19) of methylated cytosines is given. ${ }^{*}=$ target sites that overlap with tRNA locations. (d) The proportion of miCLIP-identified targets occurring in exons/introns/UTRs of mRNAs, including those sites overlapping with tRNA genes. (e) Genome browser view of the miCLIP-identified SHF mRNA methylation. Predicted methylation sites are indicated by the black peaks. Note that methylation may occur either in an SHF mRNA intron or within a tRNA, which is transcribed from the same genomic DNA strand. (f) miCLIP data showing identified sites within the CTC1 3' UTR. The CTC1 3' UTR contains three tRNA genes predicted to be transcribed from the same genomic DNA strand. The miCLIP predicted methylation sites (black peaks) overlapped specifically with these three regions. (g) An example showing that all miCLIP sequence reads, indicated by purple bars, only extend slightly beyond the annotated tRNA genes. It is most likely that these represent extensions only into the $3^{\prime}$ trailer sequences of tRNAs, and that tRNA rather than mRNA methylation is being detected.

comprehensive identification of high-confidence methylation sites.

\section{Methylation of mRNA sites that overlap with tRNA genes}

tRNA and tRNA-like sequences are sometimes found embedded within the intronic sequence of an mRNA, or more rarely in its UTR regions. The significance of this is unknown, but in addition to being present in the premRNA/mRNA sequence, it is also possible that these tRNA sequences are independently transcribed into functionally mature tRNAs. Our analysis showed that $\mathrm{m}^{5} \mathrm{C}$ sites mapping to commonly identified mRNAs can overlap with the genomic location of tRNA genes (Figure 2D). We determined how often miCLIP-identified mRNA methylation sites overlapped with the presence of tRNA genes. Most miCLIP-identified mRNA $\mathrm{m}^{5} \mathrm{C}$ sites occurred within exons, fewer within introns and more rarely in $5^{\prime}$ and 3' UTRs (Figure 2D). Of those, the vast majority of $\mathrm{m}^{5} \mathrm{C}$ sites did not coincide with the occurrence of tRNA or tRNA-like sequences, and only five miCLIP-identified mRNAs contained tRNA sequences (Figure 2D). For example, Aza-IP identified SHF as the only statistically significant mRNA containing an NSun2-dependent $\mathrm{m}^{5} \mathrm{C}$ site [23], and this site was also identified by miCLIP (Figure 2C) [24]. However, the $\mathrm{m}^{5} \mathrm{C}$ sites occurred in introns of the SHF mRNA and overlap with tRNA sequences (Figure 2E).

To address the question of whether methylation is taking place in the pre-mRNA/mRNA sequence or an independently transcribed tRNA, we focused on the $\mathrm{m}^{5} \mathrm{C}$ site within the CTS telomere maintenance complex component 1 (CTC1) mRNA as an example. The CTC1 $\mathrm{m}^{5} \mathrm{C}$ sites, one of which was also detected by bisulfite sequencing (Figure 2C), localized to the 3' UTR of the mRNA (Figure 2F). We asked whether the miCLIP sequence reads extended further into the mRNA sequence beyond the tRNA regions. If the sequence reads did not extend further into the mRNA sequence, but were confined to the tRNA sequence, it would suggest that we had detected methylation in independently transcribed tRNAs. We found that miCLIP reads usually extended only slightly beyond the annotated tRNA genes (Figure $2 \mathrm{G}$ ). Since the annotated tRNA genes do not include $5^{\prime}$ leader or $3^{\prime}$ trailer sequences, it is most likely that these small extensions overlap with tRNA 3 ' trailer sequences, which are removed post-transcriptionally. None of the sequence reads extended beyond these tRNAtrailer sequence regions, indicating that these examples represent tRNA methylation rather than methylation of pre-mRNAs/mRNAs.

\section{Other RNA methylases}

The existence of at least six more putative $\mathrm{m}^{5} \mathrm{C}$ RNA methylases in mammals may contribute to the general modest overlap between the NSun2- and Dnmt2-specific cytosine- 5 methylation sites and the transcriptome-wide methylome $[18,23,24]$. The additional enzymes NSun1 and NSun3-7 [56] are predicted to methylate RNA based on sequence conservation of key catalytic residues. Although the substrate specificities of these enzymes are unknown, NSun1 and NSun5, in addition to NSun2, have been identified as mRNA-binding proteins [57]. The biological functions of the NSun protein family is largely unknown, although all of them are expressed during mouse embryogenesis [58]. The transcripts of NSun2-7 are enriched in the developing brain, which is consistent with a proposed role in neurocognitive development [58].

The limited information we do have about the functional roles of NSun proteins indicates an essential role in a wide range of biological processes and human diseases. NSun1 (also called NOP2 or p120) is a nucleolar protein implicated in rRNA methylation and biogenesis. It has been shown to regulate the cell cycle and is involved in tumorigenesis [59-64]. Whether the enzymatic activity of NSun1 is directly required to mediate these cellular processes remains unclear. NSun4 interacts with mitochondrial transcription termination factor 4 (MTERF4) to control mitochondrial ribosomal biogenesis and translation $[65,66]$. 
The NSUN5 gene is located in a genomic region deleted in patients with Williams-Beuren syndrome, a rare neurodevelopmental disorder [67]. Patients with Williams-Beuren syndrome display intellectual disability and developmental delay, as well as craniofacial and cardiovascular abnormalities [68]. Whether deletion of NSUN5 directly contributes to these symptoms is unknown. Mutations in the NSUN7 gene cause infertility in mice due to impaired sperm motility [69]. Nothing is known regarding the functions of NSun3 and NSun6 proteins.

Together, RNA bisulfite sequencing, $\mathrm{m}^{5} \mathrm{C}$-RIP, Aza-IP and miCLIP provide powerful tools to determine methylated substrates of all NSun protein family members. This will help to provide a more detailed and informative picture of a methylome-wide $\mathrm{m}^{5} \mathrm{C}$ landscape.

\section{Delineating the epitranscriptome: comparing $\mathrm{m}^{6} \mathrm{~A}$ and $\mathrm{m}^{5} \mathrm{C}$ modifications}

Although there are several types of RNA methylation [1], so far, transcriptome-wide approaches determining site-specific RNA methylation have only been performed for $\mathrm{m}^{6} \mathrm{~A}$ and $\mathrm{m}^{5} \mathrm{C}$. As already discussed, the $\mathrm{m}^{5} \mathrm{C}$ modification is most prominently associated with various ncRNAs, but recent studies suggest that it may also be a common modification of mRNAs [18,24]. By contrast, most $\mathrm{m}^{6} \mathrm{~A}$ sites were found in mRNAs and only rarely occurred in ncRNAs, though they were, for instance, found in various long intergenic non-coding RNAs (lincRNAs) [4]. In mRNAs, both $\mathrm{m}^{6} \mathrm{~A}$ and $\mathrm{m}^{5} \mathrm{C}$ modifications are commonly found in coding regions and UTRs rather than in introns [4,24]. Enrichment of $\mathrm{m}^{6} \mathrm{~A}$ sites is most pronounced near stop codons [3,4], whereas $\mathrm{m}^{5} \mathrm{C}$ is not enriched at stop codons. Both $\mathrm{m}^{6} \mathrm{~A}$ and $\mathrm{m}^{5} \mathrm{C}$ can occur in $3^{\prime}$ UTRs $[4,18]$, suggesting a potential role in regulating microRNA responses. It is intriguing to speculate that $\mathrm{m}^{6} \mathrm{~A}$ and $\mathrm{m}^{5} \mathrm{C}$ modifications may cooperate in regulating RNA function and this should be a focus for future studies.

A key difference between the $m^{6} \mathrm{~A}$ and $\mathrm{m}^{5} \mathrm{C}$ transcriptome-wide studies is that $\mathrm{m}^{5} \mathrm{C}$ sites in RNA were experimentally determined at the nucleotide resolution, whereas the precise locations of $\mathrm{m}^{6} \mathrm{~A}$ sites have to be computationally predicted $[3,4]$. The chemistry of bisulfite sequencing makes it unsuitable for the detection of $\mathrm{m}^{6} \mathrm{~A}$ and the lack of understanding of how the $\mathrm{m}^{6} \mathrm{~A}$ modification is catalyzed makes experimental determination at the nucleotide resolution difficult. Identification of $\mathrm{m}^{5} \mathrm{C}$ sites at nucleotide resolution relies on a covalently bound enzyme-RNA catalytic intermediate, but it is not known whether $\mathrm{m}^{6} \mathrm{~A}$ modification also occurs via the formation of a temporary covalent complex. Thus, the methods available to study $\mathrm{m}^{6} \mathrm{~A}$ and $\mathrm{m}^{5} \mathrm{C}$ RNA modifications differ technically. The two studies analyzing $\mathrm{m}^{6} \mathrm{~A}$ in the transcriptome used almost identical approaches, resulting in high similarity between two datasets $[3,4]$. The development of novel techniques to identify RNA $\mathrm{m}^{6} \mathrm{~A}$ sites may be beneficial to identify additional targets not yet detected by the RIP-seq approach. However, it is clear that the currently available techniques detecting $\mathrm{m}^{6} \mathrm{~A}$ and $\mathrm{m}^{5} \mathrm{C}$ have vastly increased our knowledge regarding RNA methylation and have provided a solid platform for functional studies of these modifications.

\section{Regulatory and biological functions of $m^{6} \mathrm{~A}$ and $\mathrm{m}^{5} \mathrm{C}$ modifications}

Little is known about $\mathrm{m}^{6} \mathrm{~A}$ RNA methyltransferases, except that they consist of several subunits and include the methyltransferase like 3 (METTL3) enzymatic component [70]. No other enzyme has been identified that catalyzes RNA $\mathrm{m}^{6} \mathrm{~A}$ modification in humans. A recent study showed that METTL3-dependent $m^{6} \mathrm{~A}$ RNA methylation in circadian transcripts is crucial for regulating their own negative transcription-translation feedback loop [71]. Thus, the authors demonstrated a novel mechanism whereby RNA methylation regulates the circadian period and the function of the biological clock.

Enzymes associated with regulating both $\mathrm{m}^{6} \mathrm{~A}$ and $\mathrm{m}^{5} \mathrm{C}$ modifications have also been associated with human diseases. Genetic polymorphisms causing upregulation of the $\mathrm{m}^{6} \mathrm{~A}$-demethylase fat mass and obesityassociated (FTO) gene are associated with increased body mass index and an increased risk of obesity [72]. In addition, Fto knockout mice exhibit impaired synaptic transmission in dopaminergic neurons [73]. Interestingly, $\mathrm{m}^{6} \mathrm{~A}$ RIP-seq analyses on the mid-brain and striatum of Fto knockout mice revealed increased levels of $\mathrm{m}^{6} \mathrm{~A}$ modification in a subset of mRNAs involved in dopamine signaling pathways. Given the intellectual disability and epilepsy phenotypes observed in NSun2deficient patients, it is intriguing to speculate that the interplay of different RNA modifications may regulate neuronal signaling pathways. For example, one possibility is that mRNA methylation may regulate the translation rates of proteins that have been associated with synaptic plasticity. This hypothesis may, at least in part, explain the memory and learning deficits associated with the loss of NSun2 [37]. In addition, spatiotemporal control of protein translation at the growth cones of terminally differentiated neurons during neuronal circuit formation is known to be an important regulator of axonal pathfinding and synapse formation. It may also be worth considering whether mRNA methylation plays a regulatory role in such events.

A regulatory role for NSun2 in protein translation is supported by the fact that we showed no correlation of potentially methylated mRNAs to their turnover or stability [24]. In addition, in testicular round spermatids, 
NSun2 is a component of the chromatoid body, which is known to be a cytoplasmic subdomain for localized protein translation control [36]. Simultaneous deletion of NSun2 and Dnmt2 impair protein synthesis and tRNA stability [22]. The systematic co-ordination of protein translation steps occur during differentiation processes of embryonic stem cells [74], and aberrant translational control may contribute to the neurodevelopmental disease phenotypes in humans lacking functional NSun2 $[19,38,39,75]$. In addition, mis-regulation of protein translation has been associated with other intellectual disability associated genes, such as fragile- $\mathrm{X}$ mental retardation 1 (FMR1) [76].

The most consistent NSun2-dependent $\mathrm{m}^{5} \mathrm{C}$ site identified in a non-tRNA is C69 in the vault RNA, vtRNA1.1 [24]. Using NSun2-deficient patient-derived fibroblasts, we showed that methylation of vtRNA1.1 influenced its processing into microRNA-like molecules, which regulated the calcium channel, voltage-dependent, gamma subunit (CACNG)7 and CACNG8 mRNAs [24]. Both CACNG7 and CACNG8 encode voltage-gated calcium channels, and genes encoding ion channels are often mutated in disorders associated with epilepsy and intellectual disability [77]. We previously observed that the NSun2 protein is expressed in Purkinje neurons of the cerebellum as well as other neurons throughout the mouse brain [38]. Mis-regulation of synaptic transmission in neurons of NSun2-deficient patients may contribute to the human disease.

\section{Conclusions}

Despite all the technical advances, further optimization of current methods is required to achieve much closer agreement of the site-specific deposition of $\mathrm{m}^{5} \mathrm{C}$, especially in the non-tRNA targets identified by each method. Only then will we have a comprehensive characterization of the global distribution of $\mathrm{m}^{5} \mathrm{C}$ in RNA that will help to further define the functional roles of this modification.

The recently adapted single-molecule, real-time (SMRT) sequencing technologies for the detection of RNA sequences, together with the successful engineering of novel reverse transcriptases more sensitive to the different RNA modifications occurring in RNAs, may enable the identification of $\mathrm{m}^{5} \mathrm{C}$ transcriptome-wide without the need of any RNA processing step, not even cDNA conversion, which is usually biased and causes the loss of information about structure and base modifications [78,79].

In summary, the current transcriptome-wide approaches for determining $\mathrm{m}^{5} \mathrm{C}$ methylation sites are furthering our understanding of this RNA modification in terms of regulatory functions and disease. Indeed, it may not be long before our expectations from understanding
RNA $\mathrm{m}^{5} \mathrm{C}$ modification matches, or even exceeds, that of DNA $\mathrm{m}^{5} \mathrm{C}$ modification.

\section{Additional file}

Additional file 1: Nucleotide-resolution positions of $\mathrm{m} 5 \mathrm{C}$ sites

detected by AzaIP, miCLIP and bisulfite sequencing.

\section{Abbreviations}

Aza-IP: 5-azacytidine-mediated RNA immunoprecipitation; CACNG: Calcium channel, voltage-dependent, gamma subunit; CTC1: CTS telomere maintenance complex component 1; FTO: Fat mass and obesity-associated; HPLC: High performance liquid chromatography; lincRNA: Long intergenic non-coding RNAs; $\mathrm{m}^{5} \mathrm{C}$ : 5-methylcytosine; $\mathrm{m}^{6} \mathrm{~A}$ : 6-methyladenosine; METTL3: Methyltransferase like 3; miCLIP: Methylation-individual nucleotide resolution crosslinking immmunoprecipitation; ncRNA: Non-coding RNA; NGS: Next-generation sequencing; RIP: RNA immunoprecipitation; SHF: Src homology 2 domain containing F; UTR: Untranslated region.

\section{Competing interests}

The authors declare that they have no competing interests.

\section{Published: 29 November 2013}

\section{References}

1. Machnicka MA, Milanowska K, Osman Oglou O, Purta E, Kurkowska M, Olchowik A, Januszewski W, Kalinowski S, Dunin-Horkawicz S, Rother KM, Helm M, Bujnicki JM, Grosjean H: MODOMICS: a database of RNA modification pathways - 2013 update. Nucleic Acids Res 2013, 41:D262-D267.

2. Bodi Z, Button JD, Grierson D, Fray RG: Yeast targets for mRNA methylation. Nucleic Acids Res 2010, 38:5327-5335.

3. Dominissini D, Moshitch-Moshkovitz S, Schwartz S, Salmon-Divon M, Ungar L, Osenberg S, Cesarkas K, Jacob-Hirsch J, Amariglio N, Kupiec M, Sorek R, Rechavi G: Topology of the human and mouse m6A RNA methylomes revealed by m6A-seq. Nature 2012, 485:201-206.

4. Meyer KD, Saletore Y, Zumbo P, Elemento O, Mason CE, Jaffrey SR: Comprehensive analysis of mRNA methylation reveals enrichment in 3' UTRs and near stop codons. Cell 2012, 149:1635-1646.

5. Suzuki MM, Bird A: DNA methylation landscapes: provocative insights from epigenomics. Nat Rev Genet 2008, 9:465-476.

6. Motorin Y, Lyko F, Helm M: 5-Methylcytosine in RNA: detection, enzymatic formation and biological functions. Nucleic Acids Res 2010, 38:1415-1430.

7. Maden BE: Locations of methyl groups in $28 \mathrm{~S}$ rRNA of Xenopus laevis and man. Clustering in the conserved core of molecule. J Mol Biol 1988, 201:289-314.

8. Noon KR, Bruenger E, McCloskey JA: Posttranscriptional modifications in $16 \mathrm{~S}$ and $23 \mathrm{~S}$ rRNAs of the archaeal hyperthermophile Sulfolobus solfataricus. J Bacterio/ 1998, 180:2883-2888.

9. Smith JE, Cooperman BS, Mitchell P: Methylation sites in Escherichia coli ribosomal RNA: localization and identification of four new sites of methylation in 23S rRNA. Biochemistry 1992, 31:10825-10834.

10. Veldman GM, Klootwijk J, de Regt VC, Planta RJ, Branlant C, Krol A, Ebel JP: The primary and secondary structure of yeast $26 \mathrm{~S}$ rRNA. Nucleic Acids Res 1981, 9:6935-6952.

11. Dubin DT, Stollar V, Hsuchen CC, Timko K, Guild GM: Sindbis virus messenger RNA: the 5'-termini and methylated residues of 26 and $42 \mathrm{~S}$ RNA. Virology 1977, 77:457-470.

12. Dubin DT, Taylor RH: The methylation state of poly A-containing messenger RNA from cultured hamster cells. Nucleic Acids Res 1975, 2:1653-1668.

13. Adams JM, Cory S: Modified nucleosides and bizarre 5 '-termini in mouse myeloma mRNA. Nature 1975, 255:28-33.

14. Desrosiers R, Friderici K, Rottman F: Identification of methylated nucleosides in messenger RNA from Novikoff hepatoma cells. Proc Natl Acad Sci U S A 1974, 71:3971-3975.

15. Furuichi Y, Morgan M, Shatkin AJ, Jelinek W, Salditt-Georgieff M, Darnell JE: Methylated, blocked 5 termini in HeLa cell mRNA. Proc Natl Acad Sci US A 1975, 72:1904-1908. 
16. Lavi S, Shatkin AJ: Methylated simian virus 40-specific RNA from nuclei and cytoplasm of infected BSC-1 cells. Proc Natl Acad Sci U S A 1975, 72:2012-2016

17. Salditt-Georgieff $M$, Jelinek W, Darnell JE, Furuichi $Y$, Morgan M, Shatkin A: Methyl labeling of HeLa cell hnRNA: a comparison with mRNA. Cell 1976, 7:227-237.

18. Squires JE, Patel HR, Nousch M, Sibbritt T, Humphreys DT, Parker BJ, Suter CM, Preiss T: Widespread occurrence of 5-methylcytosine in human coding and non-coding RNA. Nucleic Acids Res 2012, 40:5023-5033.

19. Blanco S, Kurowski A, Nichols J, Watt FM, Benitah SA, Frye M: The RNAmethyltransferase Misu (NSun2) poises epidermal stem cells to differentiate. PLoS Genet 2011, 7:e1002403.

20. Brzezicha B, Schmidt M, Makalowska I, Jarmolowski A, Pienkowska J, Szweykowska-Kulinska Z: Identification of human tRNA:m5C methyltransferase catalysing intron-dependent $\mathrm{m} 5 \mathrm{C}$ formation in the first position of the anticodon of the pre-tRNA Leu (CAA). Nucleic Acids Res 2006, 34:6034-6043.

21. Frye M, Watt FM: The RNA methyltransferase Misu (NSun2) mediates Myc-induced proliferation and is upregulated in tumors. Curr Biol 2006, 16:971-981

22. Tuorto F, Liebers R, Musch T, Schaefer M, Hofmann S, Kellner S, Frye M, Helm M, Stoecklin G, Lyko F: RNA cytosine methylation by Dnmt2 and NSun2 promotes tRNA stability and protein synthesis. Nat Struct Mol Biol 2012, 19:900-905.

23. Khoddami V, Cairns BR: Identification of direct targets and modified bases of RNA cytosine methyltransferases. Nat Biotechnol 2013, 31:458-584.

24. Hussain S, Sajini AA, Blanco S, Dietmann S, Lombard P, Sugimoto Y, Paramor M, Gleeson JG, Odom DT, Ule J, Frye M: NSun2-mediated cytosine-5 methylation of vault noncoding RNA determines its processing into regulatory small RNAs. Cell Rep 2013, 4:255-261.

25. Chen Y, Sierzputowska-Gracz H, Guenther R, Everett K, Agris PF: 5-Methylcytidine is required for cooperative binding of $\mathrm{Mg} 2+$ and a conformational transition at the anticodon stem-loop of yeast phenylalanine tRNA. Biochemistry 1993, 32:10249-10253.

26. Motorin Y, Helm M: tRNA stabilization by modified nucleotides. Biochemistry 2010, 49:4934-4944.

27. Alexandrov A, Chernyakov I, Gu W, Hiley SL, Hughes TR, Grayhack EJ, Phizicky EM: Rapid tRNA decay can result from lack of nonessential modifications. Mol Cell 2006, 21:87-96.

28. Chernyakov I, Whipple JM, Kotelawala L, Grayhack EJ, Phizicky EM: Degradation of several hypomodified mature tRNA species in Saccharomyces cerevisiae is mediated by Met22 and the $5^{\prime}-3$ exonucleases Rat1 and Xrn1. Genes Dev 2008, 22:1369-1380.

29. Schaefer M, Pollex T, Hanna K, Tuorto F, Meusburger M, Helm M, Lyko F: RNA methylation by Dnmt2 protects transfer RNAs against stressinduced cleavage. Genes Dev 2010, 24:1590-1595.

30. Chow CS, Lamichhane TN, Mahto SK: Expanding the nucleotide repertoire of the ribosome with post-transcriptional modifications. ACS Chem Biol 2007, 2:610-619.

31. Warren L, Manos PD, Ahfeldt T, Loh YH, Li H, Lau F, Ebina W, Mandal PK, Smith ZD, Meissner A, Daley GQ, Brack AS, Collins JJ, Cowan C, Schlaeger TM, Rossi DJ: Highly efficient reprogramming to pluripotency and directed differentiation of human cells with synthetic modified mRNA. Cell Stem Cell 2010, 7:618-630.

32. Zhang $X$, Liu Z, Yi J, Tang $H$, Xing J, Yu M, Tong T, Shang $Y$, Gorospe $M$, Wang W: The tRNA methyltransferase NSun2 stabilizes p16INK(4) mRNA by methylating the 3'-untranslated region of p16. Nat Commun 2012, 3:712.

33. Chan $C T$, Pang YL, Deng W, Babu IR, Dyavaiah M, Begley TJ, Dedon PC: Reprogramming of tRNA modifications controls the oxidative stress response by codon-biased translation of proteins. Nat Commun 2012, 3:937.

34. Rai K, Chidester S, Zavala CV, Manos EJ, James SR, Karpf AR, Jones DA, Cairns BR: Dnmt2 functions in the cytoplasm to promote liver, brain, and retina development in zebrafish. Genes Dev 2007, 21:261-266.

35. Wei CM, Gershowitz A, Moss B: Methylated nucleotides block 5 'terminus of HeLa cell messenger RNA. Cell 1975, 4:379-386.

36. Hussain S, Tuorto F, Menon S, Blanco S, Cox C, Flores JV, Watt S, Kudo NR, Lyko F, Frye M: The mouse cytosine-5 RNA methyltransferase NSun2 is a component of the chromatoid body and required for testis differentiation. Mol Cell Biol 2013, 33:1561-1570.
37. Abbasi-Moheb L, Mertel S, Gonsior M, Nouri-Vahid L, Kahrizi K, Cirak S, Wieczorek D, Motazacker MM, Esmaeeli-Nieh S, Cremer K, Weißmann R, Tzschach A, Garshasbi M, Abedini SS, Najmabadi H, Ropers HH, Sigrist SJ, Kuss AW: Mutations in NSUN2 cause autosomal-recessive intellectual disability. Am J Hum Genet 2012, 90:847-855.

38. Khan MA, Rafiq MA, Noor A, Hussain S, Flores JV, Rupp V, Vincent AK, Malli R, Ali G, Khan FS, Ishak GE, Doherty D, Weksberg R, Ayub M, Windpassinger C, Ibrahim S, Frye M, Ansar M, Vincent JB: Mutation in NSUN2, which encodes an RNA methyltransferase, causes autosomal-recessive intellectual disability. Am J Hum Genet 2012, 90:856-863.

39. Martinez FJ, Lee JH, Lee JE, Blanco S, Nickerson E, Gabriel S, Frye M, Al-Gazali L, Gleeson JG: Whole exome sequencing identifies a splicing mutation in NSUN2 as a cause of a Dubowitz-like syndrome. J Med Genet 2012, 49:380-385.

40. Fahiminiya S, Almuriekhi M, Nawaz Z, Staffa A, Lepage P, Ali R, Hashim L, Schwartzentruber J, Abu Khadija K, Zaineddin S, Gamal H, Majewski J, Ben-Omran T: Whole exome sequencing unravels disease-causing genes in consanguineous families in Qatar. Clin Genet 2013, : . doi: 10.1111/ cge.12280.

41. Hussain S, Benavente SB, Nascimento E, Dragoni I, Kurowski A, Gillich A, Humphreys P, Frye M: The nucleolar RNA methyltransferase Misu (NSun2) is required for mitotic spindle stability. J Cell Biol 2009, 186:27-40.

42. Sakita-Suto S, Kanda A, Suzuki F, Sato S, Takata T, Tatsuka M: Aurora-B regulates RNA methyltransferase NSUN2. Mol Biol Cell 2007, 18:1107-1117.

43. Frye M, Dragoni I, Chin SF, Spiteri I, Kurowski A, Provenzano E, Green A, Ellis IO, Grimmer D, Teschendorff A, Zouboulis CC, Caldas C, Watt FM: Genomic gain of 5 p15 leads to over-expression of Misu (NSUN2) in breast cancer. Cancer Lett 2009, 289:71-80.

44. Schaefer M, Pollex T, Hanna K, Lyko F: RNA cytosine methylation analysis by bisulfite sequencing. Nucleic Acids Res 2009, 37:e12

45. Edelheit S, Schwartz S, Mumbach MR, Wurtzel O, Sorek R: Transcriptomewide mapping of 5-methylcytidine RNA modifications in bacteria, archaea, and yeast reveals m(5)C within archaeal mRNAs. PLoS Genet 2013, 9:e1003602.

46. Frommer M, McDonald LE, Millar DS, Collis CM, Watt F, Grigg GW, Molloy $\mathrm{PL}$, Paul $\mathrm{CL}$ : A genomic sequencing protocol that yields a positive display of 5-methylcytosine residues in individual DNA strands. Proc Natl Acad SCi US A 1992, 89:1827-1831.

47. Wu JC, Santi DV: Kinetic and catalytic mechanism of Hhal methyltransferase. J Biol Chem 1987, 262:4778-4786.

48. Liu Y, Santi DV: m5C RNA and m5C DNA methyl transferases use different cysteine residues as catalysts. Proc Natl Acad Sci U S A 2000, 97:8263-8265.

49. Santi DV, Garrett CE, Barr PJ: On the mechanism of inhibition of DNAcytosine methyltransferases by cytosine analogs. Cell 1983, 33:9-10.

50. King MY, Redman KL: RNA methyltransferases utilize two cysteine residues in the formation of 5-methylcytosine. Biochemistry 2002, 41:11218-11225.

51. Redman KL: Assembly of protein-RNA complexes using natural RNA and mutant forms of an RNA cytosine methyltransferase. Biomacromolecules 2006, 7:3321-3326.

52. König J, Zarnack K, Rot G, Curk T, Kayikci M, Zupan B, Turner DJ, Luscombe NM, Ule J: iCLIP reveals the function of hnRNP particles in splicing at individual nucleotide resolution. Nat Struct Mol Biol 2010, 17:909-915.

53. Behm-Ansmant I, Helm M, Motorin Y: Use of specific chemical reagents for detection of modified nucleotides in RNA. J Nucleic Acids 2011, 2011:408053.

54. Lowe TM, Eddy SR: tRNAscan-SE: a program for improved detection of transfer RNA genes in genomic sequence. Nucleic Acids Res 1997, 25:955964.

55. Chan PP, Lowe TM: GtRNAdb: a database of transfer RNA genes detected in genomic sequence. Nucleic Acids Res 2009, 37:D93-D97.

56. Reid R, Greene PJ, Santi DV: Exposition of a family of RNA m(5)C methyltransferases from searching genomic and proteomic sequences. Nucleic Acids Res 1999, 27:3138-3045.

57. Castello A, Fischer B, Eichelbaum K, Horos R, Beckmann BM, Strein C, Davey NE, Humphreys DT, Preiss T, Steinmetz LM, Krijgsveld J, Hentze MW: Insights into RNA biology from an atlas of mammalian mRNA-binding proteins. Cell 2012, 149:1393-1406.

58. Chi L, Delgado-Olguin P: Expression of NOL1/NOP2/sun domain (Nsun) RNA methyltransferase family genes in early mouse embryogenesis. Gene Expr Patterns 2013, 13:319-327. 
59. de Beus E, Brockenbrough JS, Hong B, Aris JP: Yeast NOP2 encodes an essential nucleolar protein with homology to a human proliferation marker. J Cell Biol 1994, 127:1799-1813.

60. Hong B, Brockenbrough JS, Wu P, Aris JP: Nop2p is required for pre-rRNA processing and $60 \mathrm{~S}$ ribosome subunit synthesis in yeast. Mol Cell Biol 1997, 17:378-388.

61. Gustafson WC, Taylor CW, Valdez BC, Henning D, Phippard A, Ren Y, Busch $H$, Durban E: Nucleolar protein p120 contains an arginine-rich domain that binds to ribosomal RNA. Biochem J 1998, 331:387-393.

62. Freeman JW, Busch RK, Gyorkey F, Gyorkey P, Ross BE, Busch H: Identification and characterization of a human proliferation-associated nucleolar antigen with a molecular weight of 120,000 expressed in early G1 phase. Cancer Res 1988, 48:1244-1251.

63. Ochs RL, Reilly MT, Freeman JW, Busch H: Intranucleolar localization of human proliferating cell nucleolar antigen p120. Cancer Res 1988, 48:6523-6529.

64. Perlaky L, Valdez BC, Busch RK, Larson RG, Jhiang SM, Zhang WW, Brattain $\mathrm{M}$, Busch $\mathrm{H}$ : Increased growth of $\mathrm{NIH} / 3$ T3 cells by transfection with human p120 complementary DNA and inhibition by a p120 antisense construct. Cancer Res 1992, 52:428-436.

65. Cámara Y, Asin-Cayuela J, Park CB, Metodiev MD, Shi Y, Ruzzenente B, Kukat C, Habermann B, Wibom R, Hultenby K, Franz T, Erdjument-Bromage H, Tempst $P$, Hallberg BM, Gustafsson CM, Larsson NG: MTERF4 regulates translation by targeting the methyltransferase NSUN4 to the mammalian mitochondrial ribosome. Cell Metab 2011, 13:527-539.

66. Spåhr H, Habermann B, Gustafsson CM, Larsson NG, Hallberg BM: Structure of the human MTERF4-NSUN4 protein complex that regulates mitochondrial ribosome biogenesis. Proc Natl Acad Sci U S A 2012 109:15253-15258.

67. Doll A, Grzeschik KH: Characterization of two novel genes, WBSCR20 and WBSCR22, deleted in Williams-Beuren syndrome. Cytogenet Cell Genet 2001, 95:20-27.

68. Martens MA, Wilson SJ, Reutens DC: Williams syndrome: a critical review of the cognitive, behavioral and neuroanatomical phenotype. J Child Psychol Psychiatry 2008, 49:576-608.

69. Harris T, Marquez B, Suarez S, Schimenti J: Sperm motility defects and infertility in male mice with a mutation in Nsun7, a member of the Sun domain-containing family of putative RNA methyltransferases. Biol Reprod 2007, 77:376-382.

70. Bokar JA, Shambaugh ME, Polayes D, Matera AG, Rottman FM: Purification and cDNA cloning of the AdoMet-binding subunit of the human mRNA (N6-adenosine)-methyltransferase. RNA 1997, 3:1233-1247.

71. Fustin JM, Doi M, Yamaguchi Y, Hida H, Nishimura S, Yoshida M, Isagawa T, Morioka MS, Kakeya H, Manabe I, Okamura H: RNA-methylation-dependent RNA processing controls the speed of the circadian clock. Cell 2013, 155:793-806.

72. Church C, Moir L, McMurray F, Girard C, Banks GT, Teboul L, Wells S, Brüning JC, Nolan PM, Ashcroft FM, Cox RD: Overexpression of Fto leads to increased food intake and results in obesity. Nat Genet 2010 , 42:1086-1092.

73. Hess ME, Hess S, Meyer KD, Verhagen LA, Koch L, Brönneke HS, Dietrich MO, Jordan SD, Saletore Y, Elemento O, Belgardt BF, Franz T, Horvath TL, Rüther $U$, Jaffrey SR, Kloppenburg P, Brüning JC: The fat mass and obesity associated gene (Fto) regulates activity of the dopaminergic midbrain circuitry. Nat Neurosci 2013, 16:1042-1048.

74. Ingolia NT, Lareau LF, Weissman JS: Ribosome profiling of mouse embryonic stem cells reveals the complexity and dynamics of mammalian proteomes. Cell 2011, 147:789-802.

75. Kuss AW, Garshasbi M, Kahrizi K, Tzschach A, Behjati F, Darvish H, AbbasiMoheb L, Puettmann L, Zecha A, Weissmann R, Hu H, Mohseni M, Abedini SS, Rajab A, Hertzberg C, Wieczorek D, Ullmann R, Ghasemi-Firouzabadi S, Banihashemi S, Arzhangi S, Hadavi V, Bahrami-Monajemi G, Kasiri M, Falah M, Nikuei P, Dehghan A, Sobhani M, Jamali P, Ropers HH, Najmabadi H: Autosomal recessive mental retardation:homozygosity mapping identifies 27 single linkage intervals, at least 14 novel loci and several mutation hotspots. Hum Genet 2011, 129:141-148.

76. Darnell JC, Van Driesche SJ, Zhang C, Hung KY, Mele A, Fraser CE, Stone EF, Chen C, Fak JJ, Chi SW, Licatalosi DD, Richter JD, Darnell RB: FMRP stalls ribosomal translocation on mRNAs linked to synaptic function and autism. Cell 2011, 146:247-261.
77. Poduri A, Lowenstein D: Epilepsy genetics - past, present, and future. Curr Opin Genet Dev 2011, 21:325-332.

78. Roberts RJ, Carneiro MO, Schatz MC: The advantages of SMRT sequencing. Genome Biol 2013, 14:405.

79. Vilfan ID, Tsai YC, Clark TA, Wegener J, Dai Q, Yi C, Pan T, Turner SW, Korlach $\mathrm{J}$ : Analysis of RNA base modification and structural rearrangement by single-molecule real-time detection of reverse transcription. $J$ Nanobiotechnol 2013, 11:8.

doi:10.1186/gb4143

Cite this article as: Hussain et al: Characterizing 5-methylcytosine in the mammalian epitranscriptome. Genome Biology 2013 14:215. 